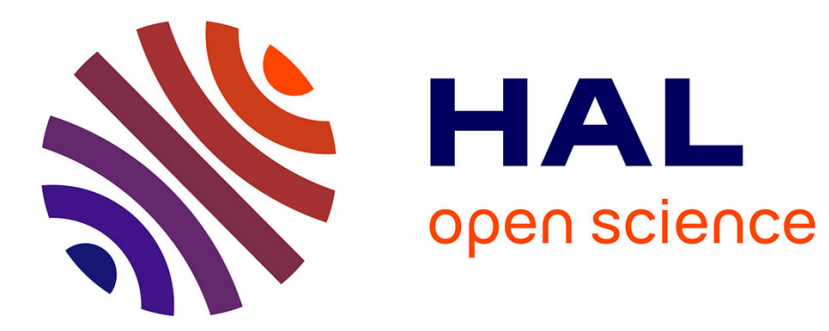

\title{
Louis Dumont et la science indigène
}

Roland Lardinois

\section{To cite this version:}

Roland Lardinois. Louis Dumont et la science indigène. Actes de la Recherche en Sciences Sociales, 1995, 106 (1), pp.11-26. 10.3406/arss.1995.3132 . halshs-02005120

\section{HAL Id: halshs-02005120 \\ https://shs.hal.science/halshs-02005120}

Submitted on 3 Feb 2019

HAL is a multi-disciplinary open access archive for the deposit and dissemination of scientific research documents, whether they are published or not. The documents may come from teaching and research institutions in France or abroad, or from public or private research centers.
L'archive ouverte pluridisciplinaire HAL, est destinée au dépôt et à la diffusion de documents scientifiques de niveau recherche, publiés ou non, émanant des établissements d'enseignement et de recherche français ou étrangers, des laboratoires publics ou privés. 


\section{Monsieur Roland Lardinois}

\section{Louis Dumont et la science indigène}

In: Actes de la recherche en sciences sociales. Vol. 106-107, mars 1995. Histoire sociale des sciences sociales. pp. 11-26.

Citer ce document / Cite this document :

Lardinois Roland. Louis Dumont et la science indigène. In: Actes de la recherche en sciences sociales. Vol. 106-107, mars 1995. Histoire sociale des sciences sociales. pp. 11-26.

doi : 10.3406/arss.1995.3132

http://www.persee.fr/web/revues/home/prescript/article/arss_0335-5322_1995_num_106_1_3132 


\title{
Roland Lardinois
}

\section{LOUIS DUMONT ET LA SCIENCE INDIGÈNE}

\begin{abstract}
On a parmi nous suivi [les] errements (des hindousl avec une docilité fâcheuse. J'entends surtout les indianistes. Représentants de l'école philologique, ils obéissent à une pente presque irrésistible l...l. La théorie brâhmanique est comme leur atmosphère propre.
\end{abstract}

Émile SFAART, Les Castes dans l'Inde, 1894.

PARTIR DES A.NéES 1950, les recherches menées par Louis Dumont sur l'organisation sociale des castes, sur le panthéon hindou ou sur l'Inde ancienne ont profondément renouvelé les études indiennes. Ces recherches révèlent en effet une pratique novatrice de l'anthropologie, soucieuse d'éclairer les faits observés sur le terrain par la connaissance des textes sanscrits qu'étudient les indologues: publié à la fin des années 1960, Homo bierarchicus ${ }^{1}$, dans lequel Dumont expose de manière méthodique son modèle de compréhension du monde hindou, témoigne de ce renouvellement. On ne saurait cependant comprendre l'anthropologie dumontienne sans prendre en compte aussi les Essais sur l'individualisme $^{2}$ : dans ces travaux, Dumont se donne pour

1 - L. Dumont, Homo hierarchicus. Fisai sur le système des castes, Paris, Gallimard, 1966, rééd., Gallimard, coll. "Tel", 1979. Cette réédition est augmentée d'une préface clans laquelle Louis Dumont fait le point sur les débats suscités par l'ouvrage. et dune postface dans lacquelle il expose sa thérorie de la hiérarchie; toutes les références renvoient a celte réedition (les termes sanscrits sont translittérés sans signe diacritique).

2 - I. I) umont, Essais sur l'inditidualisme. Ine perspective anthropolegique sur l'idéologie moderne, Paris, Éd. du Seuil, 1983.

\section{Pourquoi j'ai choisi l'Inde? J'ai lu très tôt René Guénon*}

Pour choisir l'Inde, l'enseignement de Mauss aurait suffi, avec le sentiment que la perspective chronologique extrêmement décevante appliquée par les philologues à la compréhension de la civilisation indienne demandait à être complétée, voire supplantée, par une perspective sociologique appliquée d'abord à l'observation et à la compréhension du présent, puis extrapolée vers le passé.

En fait, une influence lointaine, beaucoup plus ancienne, a certainement joué. Grâce à Roger Caillois, j'avais fréquenté quelque temps le petit groupe du «Grand Jeu», soit, à l'époque, René Daumal et Roger Gilbert-Lecomte. Sous leur influence, je lus sur l'Inde René Guénon, pour qui elle était une sorte de paradis de la "Tradition », une société parfaitement immobile, en possession sereine des secrets ultimes de l'Être. Plus tard, quand je me préparai à enquêter dans l'Inde et que j'y partis effectivement, j'étais certes très loin de penser encore à Guénon, et préoccupé de choses beaucoup plus terre à terre pour commencer. Mais un beau jour, encore beaucoup plus tard, je me rendis compte qu'avec mon article sur le renoncement, qui voulait donner une vue d'ensemble des religions de l'Inde, j'avais pris presque exactement le contrepied des thèses de Guénon. N'avais-je pas, en effet, là où il pensait avoir définitivement exorcisé l'individualisme, trouvé dans le renonçant, c'est-à-dire dans l'individu hors du monde, le grand «créateur de valeur » du monde indien? Je raconte cela pour faire voir la limite des «influences», la distance qu'il peut y avoir entre un premier éveil de la curiosité et ce à quoi on aboutit finalement'.

* Entretien avec Louis Dumont, Revue européenne des sciences sociales, XXII, 1984, 68, p. 157.

I - «Dumont l'intouchable » (entretien avec Jean-Paul Enthoven), Le Nouvel Observateur, 6 janvier 1984. 
tâche de penser la spécificité du monde occidental centré sur les valeurs égalitaires qu il oppose aux valeurs hiérarchiques prévalant dans le monde inclien et, plus généralement, dans les univers dits traditionnels ou prémodernes.

Cette ceuvre a suscité d'amples controverses. Se fondant sur une analyse interne, nombre d'anthropologues ont exprimé depuis longtemps leur désaccord avec un point de vue sur le monde indien que l'on peut qualifier de "brahmanocentrique" ${ }^{3}$. Plus récemment, des historiens ont développé une critique externe de l'ceuvre dans laquelle ils suspectent l'anthropologie dumontienne d'être "lhéritière manifeste du legs orientaliste" colonial ${ }^{4}$. Ces critiques soulèvent au moins un paradoxe quielles semblent toutes ignorer: comment le point de vue brahmanique peut-il se substituer au point de vue savant dans la compréhension du système des castes de Dumont, alors même que celui-ci se réclame à juste titre de la sociologie durkheimienne, qui opère précisément la distinction entre compréhension indigène et compréhension sociologique?

pour répondre à cette question, il faut prendre au séricux Louis I Dumont lorsqu il affirme devoir son intérêt pour l'Inde non seulement à l'enseignement de Marcel Mauss mais aussi à la lecture qu il fit " très tôt ", au clébut des années 1930, de l'essayiste ésotériste René Guénon. La lecture comparée de ces deux auteurs suggère en effet plus qu'une simple "influence" de Guénon sur l'ceuvre de l)umont ${ }^{5}$. Dans ce travail, on s'efforcera de rendre compte, à la fois, de l'unité des principes d'intelligibilité du monde social que l'anthropologie dumontienne développe et de ce que ces principes doivent à leur espace social de production, c'est-à-dire au champ français des études indiennes entendu comme espace de médiation objectif des déterminations externes, retraduites dans l'xuvre en termes de problématiques et de méthodes, de théories et de concepts, bref en choix scientifiques.

\section{«ORIENT ET OCCIDENT »: UNE PENSÉE DE L'ORDRE ET DE LA HIÉRARCHIE}

En 1921, René Guénon publie son premier livre sur l'hindouisme, Introduction générale à l'étude des doctrines hindontes ${ }^{6}$, qui marque le début d'une carrière d'essayiste dont la production se définit, en gros, par une double orientation : d'une part, la défense de la tradition et des grands courants initiatiques et, d'autre part, la critique du monde moderne et du développement de l'individualisme occidental. Mais l'originalité de Guénon tient au fait qu'il défend un point de vue comparatif qui consiste à éclairer le monde moderne à la lumière des grandes civilisations orientales.

On peut définir Guénon comme un philosophe de l'ésotérisme doctrinal ${ }^{7}$ qui se conne pour tâche d'exposer la "tradition", c'est-à-dire les principes métaphysiques "immuables et éternels " qui fondent les relations des hommes entre eux et avec le monde socio-cosmique : il s'agit pour lui de saisir ce monde comme totalité signifiante, comme un "Tout universel " qui exprime des principes d'ordre transcendant "supra-individucl " et "supra-rationnel ". Ces principes constituent, selon Guénon, la "tradition primordiale", le fonds commun de l'humanité, son état "normal". Mais cette "tradition primordiale " ne s'exprime à chaque fois que dans des cas particuliers qui sont autant d'" applications contingentes " de la tradition dont l'Inde représente l'archétype. Dans cette vision du monde, la civilisation occidentale représente pour Guénon une rupture à la fois particulière et unique par laquelle "l'esprit moderne "sest constitué contre "l'esprit traditionnel ", sous l'emprise du travail critique de la raison. Cette opposition archétypale entre la "tradition" et la "modernité" se confond ici avec l'opposition entre "Orient et Occident".

La connaissance de l'hindouisme, selon Guénon, implique au moins deux postulats explicites. Premièrement, Guénon condamne formellement toute appréhension historique de l'hindouisme, au nom du caractère immuable, éternel des "principes" qui fondent la tradition : ceux-ci sont de nature métaphysique et ne peuvent s'étudier que métaphysiquement ${ }^{8}$. Deuxièmement, il affirme la supériorité absolue du point de vue indigène sur "le point de vue occidental " dans toute entreprise de

3 - Cf. par exemple. R. Burghart, "Ethnographers and their Local Counterparts in India ", in R. Fardon (ed.). Localizing Strategies. Regional Traditions of Ethnographic Writing. Fdimbourg, Scottish Academic Press. Washington, Smithsonian Institution, 1990, p. 260-279.

4 - C. A. Breckenridge et P. van der Veer (eds). Orientalism and the Pestcolomial Predicament, Philadelphie, Lniversity of Pennsylvania Press, 1993 , p. 13

$5-\grave{A}$ propos de Guénon, "ne parlons pas trop d’influence " concède aujourd hui encore Louis Dumont en la reconnaissant spontanément (entretien personnel avec Louis Dumont, 25 mars 1993).

6-R. Guénon. Introduction générale à l'étude des doctrines bindoues. Paris, Librairic Marcel Rivière, 1921. Outre cet ouvrage, on peut citcr Orient et Occident, Paris, Guy Trédaniel, 1987; I.bomme et son devenir selon le Vedânta, Paris, Éd. traditionnelles, 1982: La Crise du monde moderne. Paris, Gallimard, 1946. Sauf précision, les citations renvoient à ces ouvrages.

7 - Sur l'ésotérisme en général et la place qu'y occupe René Guénon, cf. A. Faivre, Accés de l'ésotérisme occidental, Paris, Gallimard, 1986.

8-R. Guénon, Introduction générale à létude des doctrines hindrues. ()p. cit., p. 100. 
connaissance de la tradition : les brahmanes sont pour lui les "interprètes autorisés" " de la tradition et il suffit de se faire les porte-parole de ceux-ci pour exprimer la vérité de cette tradition.

Pour comprendre les conséquences que les thèses guénoniennes ont eues sur la connaissance savante de lhindouisme, il faut revenir sur un petit opuscule publié à la fin des années 1920, Autorité spirituelle et Pouvoir temporel ${ }^{10}$. Dans cette brochure, Guénon expose de manière très claire sa conception du modèle universel de la relation hiérarchique selon laquelle le pouvoir temporel du roi est englobé dans l'autorité spirituclle du prêtré. Dans le monde hindou. "l'autorité des brahmanes" incarne un "principe d'unité supérieure" qui fonde l'ordre social en le légitimant, de sorte que le pouvoir temporel des ksatriva (guerriers) ne peut que lui être normalement "subordonné". Dans cette civilisation "où la religion pénètre toute l'existence de l'être humain ", le pouvoir politique et "tout ce qui constitue [...] la vie sociale se trouve comme englobée dans son clomaine ". À partir de cette théorie brahmanique du pouvoir, exposée on ne peut plus sommairement par Guénon, celui-ci développe un point de vue organiciste selon lequel il y a une correspondance parfaite et harmonieuse entre la hiérarchie spirituelle des principes et la hiérarchie sociale des castes: celles-ci sont ordonnées selon leur "nature" et leur "fonction" traditionnelles dans lordre du monde quiexprime la notion de dharma ${ }^{12}$.

Le second thème de réflexion qui donne pleinement son sens à l'oxuvre de Guénon est la critique que cet auteur ne cesse dadresser à toutes les formes de la modernité occidentale qui mettent en question les fondements des univers sociaux traditionnels: il s'agit pour l'essentiel de lindividualisme et du travail critique de la raison et de la science, autant de thèmes que Guénon développe dans la Crise du monde moderne. Cette partie de l'xuvre de Guénon est une tentative pour esquisser, de son point de vue, une genèse de la modernité et de loccidentalisme occidentaux. Ainsi, la "modernité" apparât-elle pour cet auteur comme une "cléviation et une anomalie " par rapport à la "tradition primordiale". Ft la Révolution française marque l'aboutissement d'une longue évolution, le point de rupture brutal avec lancien ordre traditionnel "normal". La "science moderne", selon Guénon, est partic prenante de ce processus de rupture et de désacralisation du monde enchanté passé. Parce qui elle est de "caractère tout "empirique" 13." cette "science profance représente une version appauvrie de la "science sacrée" traditionnelle, dont elle ne conserve en son sein que des "résidus" ".t. Aussi, Guénon se propose-t-il dinverser les points de vue : cest en se fondant sur les principes de la tradition que lon peut "saisir la vraie signification du monde moderne" puisque, pour comprendre celui-ci, il faut " n'en être affecté à aucun degré " ${ }^{15}$. Guénon se donne alors pour tâche de travailler a une "restauration" de la tradition et de la "science sacrée" dans une ceurre essentiellement polémique. au caractère à la fois illuminé et prophétique. et qui se situe à l'opposé de tout travail savant de type historique ou sociologique.

\section{René Guénon: une trajectoire d'eXclu}

Dans l'entre-deux-guerres, la trajectoire de Guénon est définice par un double rejet: d'une part. celui des indianistes de l'Université et, d autre part, celui des milieux intellectucls de l'Institut catholique de Paris. Ce double rejet rend compte de la position dominée et marginale que Guénon occupe dans l'univers des études indiennes et, plus généralement, dans le champ intellectuel cles années 1930.

René Guénon est né à Blois en 1886 dans une famille catheslique pratiquante de la moyenne bourgeosisie provinciale: son père était architecte-expert dans un cabinet d'assurances. Après des études secondaires dans lenseignement privé à Blosis. René Guénon commence à Parris une classe préparatoire à l'école polytechnique qu'il abandenne en cours dannée. Il s'inscrit a la Faculté des lettres de Paris et obtient un diplôme d'études supérieures de philossphie en 1916. mais il échoue à lagrégation trois ans plus tard. Il exerce alors comme enseignant de phikesophie dans divers établissements publics et privés avant de quitter définitivement la France en 1930) pour le Caire, ou il meurt. converti à lislam, en $1951^{16}$.

Au début des années 1920, Guénon souhaitait soutenir une thèse sur la philosophie hindoue avec sylyain

$9-$ Ibid. p. 52.

$10-$ R. Guénon. Autorite spirituelle et Poutoir temponel. Paris. Ciuy Trédaniel. 198:

$11-$ R. Guénon, Hélanges, Paris. Gallimard. 19)-6. p. T2 (je soulignes).

12 - La notion de abarma désigne at la fois fordre socio-cosmicpue comme principe transcendant el les devoirs que chaque hinctou. selon son groupe dappartenance. est socialement contraint dienererer pour contribuer au maintien de cel ordre.

13 - R. Guénon. Ie Regne de la chuantite et le signe des temps. Paris (allimard. $1972\left(1^{\mathrm{re}}\right.$ ed. 19+5). p. - + (je sesuligne)

1.4 - Ibid. en particulier p. 180)-186 (je souligne): sur la criticjue de la science of. Orient et occident. op). cit.. en particulier p. $+1-3.3$

15 - R. Guénon. le Regne de la quantite et le signe des temps. Paris. (iallimard. 19-2. p. 8 .

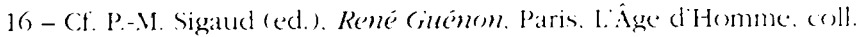
Les dossiers H . 1984. p. 299-301 
Lévi ${ }^{-}$, qui dominait alors, par son savoir et ses positions académiques, lindianisme français. Mais ce dernier, qui avait d'abord accepté le sujet, refusa que Guénon soutienne sa thèse, jugeant son travail inacceptable du point de vue académique: "Il entend exclure tous les éléments qui ne correspondent pas à sa conception, écrit Sylvain Lévi dans son rapport au doyen de la Sorbonne. [...] Tout est dans le Vedânta [...]. Il fait bon marché de l'histoire et de la critique historique ${ }^{18}$. "Rejeté par les inclianistes savants, Guénon fut introduit par Jacques Maritain à l'Institut catholique de Paris, que tout opposait, socialement et intellectuellement, à l'"esprit de la nouvelle Sorbonne "19. Les philosophes néo-thomistes comme le père Peillaube, fondateur et directeur de la Revne de philosophie, et le père Sertillange, secrétaire de la Revue thomiste, se reconnaissaient pour une part dans la critique de la théosophie que Guénon avait publiée en 1921. Introduit dans plusieurs revues théologico-philosophiques liées à ces courants de pensée catholiques, Guénon développe clans ses articles un point de vue syncrétiste inspiré, d'un côté, d'un traditionalisme védanto-occultiste et, de l'autre, des formes primitives du christianisme. Mais cette tentative échoue et, en 1927, il est également exclu de ces milieux ${ }^{20}$.

Pour comprendre les raisons de ce double rejet, il faut revenir sur les débuts de la trajectoire intellectuelle de Guénon. Depuis les années 1905-1910, celui-ci fréquentait activement, au gré de ses affiliations diverses, nombre de confréries ésotériques (gnostiques, kabbalistes, rose-croix, francs-maçonnes et autres). Si ces courants saffrontaient dans des luttes doctrinales internes et externes, ils sidentifiaient tous dans une même quête diordre mystique, une même recherche de la "tradition primordiale "que l' "Occident "aurait perdue mais dont l' "Orient "serait encore le détenteur. Les doctrines syncrétiques de ces groupements intègrent des éléments divers empruntés indifféremment, entre autres, aux grandes religions de l'Asie et du Moyen-Orient, qu'il sagisse du brahmanisme d'inspiration védantique néohindoue, du taoïsme ou de lisslam. Dans ce milieu hétérogène, la particularité de Guénon tient à l'ambivalence de sa position et à la fonction d'unification syncrétique vers laquelle tend le système intellectuel qu'il développe: critiquant la théosophie et le spiritisme, dont il dénonce les simulacres, Guénon défend l'universalité d'une pensée métaphysique qui intègre les apports les plus divers des civilisations orientales dont les traditions ne lui semblent pas contradictoires avec celles du christianisme primitif.

Toutefois, on ne peut comprendre complètement les effets que produisent ces courants ésotéro-occultistes dans le champ académique, si l'on omet de s'interroger sur les relations qu'ils entretiennent encore avec le champ politique dans les premières décennies de ce siècle. Les adeptes des courants de pensée qui se réclament de la tradition partagent avec nombre de philosophes néo-thomistes un même sentiment antimoderne entendu dans un double sens. Il s'agit, d'abord, d'une réaction intellectuelle et morale aux nouvelles sciences religieuses qui se sont développées dans l'Université à la fin du xix ${ }^{e}$ siècle, en particulier à l'École pratique des hautes études, et dont les méthodes de type positiviste remettaient en cause le fondement des "sciences sacrées " dont l'Église avait le monopole (c'est la querelle du modernisme catholique). Mais cet antimodernisme catholique se doublait aussi d'un antimodernisme politique, c'est-à-dire d'une critique du "démocratisme" et de l'égalité comme système de valeurs hérité de la révolution des Lumières. Autour de la Première Guerre mondiale, ces deux composantes du sentiment antimoderne se sont cristallisées dans le mouvement de l'Action française de Charles Maurras ${ }^{21}$. Pour les tenants de ces courants de pensée, la droite nationaliste incarnait dans l'ordre temporel les mêmes valeurs sociales, politiques et éthiques que l'Église défendait, pour sa part, dans le domaine spirituel. Et si Guénon n'a jamais été engagé personnellement dans ce mouvement, les thèses intellectuelles qu'il défend, ses opinions politiques autant que ses amitiés, le font appartenir pleinement à cette famille idéologique.

Mais, en 1926, la condamnation de l'Action française par le Vatican ouvre une crise profonde entre les intellectuels catholiques et le parti de Maurras. Cette crise est à l'origine de l'exclusion de Guénon des milieux néothomistes. Mais c'est également dans ce contexte polémique que Guénon rédige en 1929 son opuscule, Autorité spirituelle et Pouvoir temporel, à l'intention de ses amis qui refusent la sanction de Pie XI. Acte militant au

17 - Sylvain Lévi (1863-1935), agrégé de lettres en 1883. était notamment directeur d'études à la section des sciences religieuses de l’École pratique des hautes études et titulaire de la chaire de "Langue et littérature sanscrite "du Collège de France depuis 1894

18 - Extrait du rapport de Sylvain Lévi au doyen Brunot, cité par J.-P. Laurent, Le Sens caché dans líxulre de René Guénon, Paris, LîAge d'Homme. 1975 , p. 67.

19 - Sur cette opposition, voir le jugement que les Maritain portent sur la Sorbonne au début du siècle. in R. Maritain, Mes Grandes Amitiés, Paris, Desclée de Brouwer, 1992 (1 ${ }^{\mathrm{re}}$ éd. 1949), en particulier p. 49-89.

20 - Cf. M.-F. James, Esotérisme el Christianisme autour de René Guénon, Paris. Nouvelles Éditions latines, 1981.

21 - Sur celte rencontre, of. V. Nguyen, "Maistre, Maurras, Guénon : contre-révolution et contre-culture", in P.-M. Sigaud (ed.). René Guénom, op. cit.. p. 175-191. 
sens guénonien du terme, c'est-à-dire intervention "du point de vue purement doctrinal " "dont tout le reste dérive " 22 , et non du point de vue de l'action politique contingente, ce texte se présente comme une défense explicite de la position de l'Église au nom de l'universalité de la doctrine ou, si l'on veut, de son caractère englobant: dans cette brochure, le cas de l'Inde est convoqué, au même titre que la société médiévale occidentale, pour illustrer la suprématie universelle "du spirituel sur le temporel".

\section{LA SOCIOLOGIE DURKHEIMIENNE EN JEU}

Cependant, il faut encore comprendre comment ces luttes sociales et politiques ont été retraduites en termes de connaissance dans le champ spécialisé des études indiennes. Au tournant du siècle, le développement de la sociologie durkheimienne au sein de l'université a bouleversé la hiérarchie entre les disciplines et introduit de nouveaux objets de recherche ${ }^{23}$. I)ans l'univers des études indiennes, cette transformation s'est traduite par un infléchissement sociologique notable : l'anthropologie naissante s'est mise à l'étude des textes sanscrits pour mieux comprendre les structures sociales et cognitives du monde hindou, alors que, en retour, les disciplines classiques s"enrichissaient des interrogations de l'école française de sociologie, nourries par ailleurs des premières recherches empiriques britanniques. En montrant, notamment, que la fonction classificatrice et, plus généralement, les systèmes symboliques sont le résultat de déterminations à la fois historiques et sociales variables culturellement, la sociologie durkheimienne permettait à l'indologie classique d'entreprendre un travail de dénaturalisation des catégories collectives de la pensée brahmanique, en les arrachant à la vision essentialiste du monde social dans lesquelles elles s'enracinent.

Lorsque Hubert et Mauss publient en 1899 L'Essai sur la nature et la fonction du sacrifice, ils se fondent sur les recherches que Sylvain lévi a effectuées à leur intention sur La Doctrine du sacrifice dans les Brâbmanas, selon le titre de louvrage publié en $1898^{2 \cdot t}$. À la même époque, les premières publications anthropologiques qui accompagnent les recensements indiens suscitent deux travaux importants sur le système des castes, celui du sanscritiste Émile Senart ${ }^{25}$ et, quelques années plus tard, en 1908, celui du sociologue proche de Durkheim, Célestin Bouglé. dont le travail se fonde sur les publications censitaires coloniales ${ }^{26}$.

Cependant, au pôle opposé du champ de production indianiste se sont cristallisés des principes intellectuels de nature différente. Nayant pu faire reconnaître son point de vue ni dans l'univers de l'indianisme savant ni parmi les milieux catholiques les plus proches de ses conceptions éthico-politiques, Guénon s'est finalement imposé au sein de la mouvance ésotérique qu'il n’avait jamais réellement quittée. Au début des années 1930, il prend la direction de la revue Voile d'Isis dont le titre devient Études traditionnelles, publication privilégiée de sa pensée, qui fait école. En important dans les courants ésotériques des méthodes d'étude et des problématiques empruntées aux disciplines universitaires classiques et à l'exégèse catholique, Guénon souhaitait faire de cette publication une grande revue française du traditionalisme orientaliste ${ }^{27}$. Ainsi, à l'extrême opposé du Journal asiatique et de l'orientalisme érudit, s'est cristallisé autour de Guénon, pour une part, le pôle non universitaire (et même anti-universitaire) du champ de production indianiste ${ }^{28}$. Les principes d'ordre épistémologique qui fondent la connaissance de type guénonienne des grandes civilisations orientales sont en opposition totale avec ceux de la sociologie durkheimienne. Et Guénon $n$ 'a cessé de dénoncer dans ses écrits ce qu'il nommait, avec mépris, l' "école sociologique " ${ }^{29}$ française, dont la légitimité scientifique était alors forte au sein de la nouvelle Sorbonne. Tout opposait en effet, d'un côté, les tenants de l'université läque, républicaine et rationaliste, défenseurs d'un savoir de type positif et d'une morale émancipée de la religion, qu'étaient Marcel Mauss et

22 - R. Guénon, Autorité spirituelle et Poutoir temporel. of. cit., p. 10) et 13 .

23 - Cf., entre autres, C. Charle, La France des universitaires 1870 1940\%, Paris, Éd. du Seuil, 1994, et V. Karady, "1)urkheim, kes sciences sociales et l'tiniversité : bilan d'un semi-échec", Revue française de sociologie, XVII. 2, avril-juin 1976, p. 267-311

24 - Sur les relations entre Sylvain Lévi et Marcle Mauss, Cf. M. Fournier. Marcel Mauss, Paris. Fayard, 1994, p. 93-104 et p. 150-155.

25 - É. Senart, les Castes dans l'Inde. Ies faits et le sbsteme, Paris, Librairie orientaliste Paul Geuthner, 1927: louvrage rassemble des articles publiés en 1894.

26 - C. Bouglé, Essai sur le régime des castes. $3^{\circ}$ éd., Paris. PL F, 1935. 27 - Cf. "Lne entreprise guénonienne : "Les Éditions traditionnelles" ", Planéte plas, avril 1970, p. 141.

28 - René Guénon nincarne pas ce pôle à lui seul. Il faudrait encore prendre en compte la position de Romain Rolland et le travail de diffusion du gandhisme et des mourements réformistes néo-hindous que celui-ci a effectué dans lentre-deux-guerres. Mais par son engagement politique avec les partis de gauche. Romain Rolland se situe a l'opposé de Guénon, qui le percevait dailleurs comme l'un de ses concurrents (je remercie Charles Malamoud d'avoir attire mon attention sur la vision particulière de l'Inde que développe Romain Rolland).

29- - Cf. par exemple, R. Guénon. Orient et Occident. of) cit. p. 30): sur les luttes menées par la droite nationaliste à lencontre de I)urkheim et de la sociologie, of. W. Lepenies. Les Trois Cultures. Entre science et littérature. lavènement de la sociologie. Paris. F.d.de la Maison des sciences de lhomme, 1990, en particulier p. 45-86. 
Sylvain Lévi pour ne citer que ces deux noms et, de lautre, les cléfenseurs de la culture classique, catholique et nationaliste auxquels Guénon appartenait, contempteurs de la raison et militants d'une pensée de l'ordre et de la hiérarchie.

\section{DE «LA CRISE DU MONDE MODERNE» À L'ANTHROPOLOGIE DE LA MODERNITÉ}

Le rapprochement entre les ceuvres de René Guénon et de Louis Dumont s"impose pour rendre compte des contradictions internes que rencontre la compréhension de lanthropologie dumontienne. Mais en affirmant avoir pris "presque exactement le contre-pied des thèses de Guénon ${ }^{30}$ ", Dumont, qui reconnaitt sa dette pour mieux s'en distinguer, met le sociologue dans une position difficilement tenable, puisqu'il l'expose à être continuellement démenti. Les traces et les symptômes que l'analyse dégage apparaissent alors comme le faisceau des présomptions les plus probables permettant de rendre compte des faits conquis contre les diverses censures de l'univers indianiste, qui interdisaient de les construire méthodiquement pour les rendre visibles.

Ainsi, le projet de sociologie comparée que Dumont entend fonder, peut être compris comme la retraduction dans l'univers de l'anthropologie sociale des années postérieures à la Seconde Guerre mondiale de questions d'ordres philosophique et sociologique élaborées antérieurement et sous d'autres formes, en particulier dans les espaces dominés du champ de production des savoirs indianistes. Tout se passe en effet comme si l'(xuvre de Dumont était le résultat de la rencontre de deux espaces intellectuels et sociaux qui sont aussi deux espaces épistémologiques contradictoires, constitutifs de la structure du champ des études indiennes en France dans les années 1930: d'un côté, l'espace du courant de pensée ésotériste de la tradition rassemblé autour de René Guénon et qui, se fondant sur une philosophie néo-aristotélicienne (ou néo-thomiste dans la version développée par des agents appartenant à l'univers catholique), produit une vision essentialiste et organiciste du monde social en général et de l'Inde en particulier; de l'autre, l'espace de la sociologie durkheimienne et de l'indologie classique qui développe un savoir de type positif et rationaliste, contribuant ainsi, pour sa part, à mettre à jour les déterminations historiques et sociales des catégories de pensée du monde hindou.

30 - "Dumont l'intouchable " (entretien avec Jean-Paul Enthoven). le Nouvel obseriateur, 6 janvier 1984, repris in Revuc europeenne des sciences sociales, XXII, 1984, 68, p. 31.

\section{L'UNIVERS THÉORIQUE EXPLICITE DE L'ANTHROPOLOGIE DUMONTIENNE}

P

our comprendre le sens du projet de Dumont et mesurer l'importance des ruptures qu'il opère dans l'ordre de la connaissance du monde indien, il faut, dans un premier temps, rapporter sa démarche à son univers théorique explicite. Premièrement, s'incrivant à la fois dans la sociologie des formes symboliques esquissée par Durkheim et Mauss et dans la logique du projet structuraliste défini par Lévi-Strauss, Dumont fait de l'hindouisme un système de valeurs englobant, pan-indien, qu'il nomme idéologie; il considère en outre que ce système de valeurs fait sens et qu'il peut être isolé des autres aspects du monde social. Dumont oppose alors l'idéologie, ainsi définie, à sa « composante résiduelle empirique » (je souligne), c'est-à-dire aux faits d'ordre socio-économique. Cette opposition principale commande la seconde distinction que fait Dumont entre le système des castes considéré, d'une part, comme système de valeurs (ou idéologie) qu'exprime le modèle lettré indigène des varna et, d'autre part, comme système empirique des jati. Deuxièmement, se référant à Weber, Dumont affirme que les agents sociaux agissent "consciemment » en fonction de ce système de valeurs qui informe et oriente leurs actions'. En considérant l'hindouisme comme une forme symbolique à part entière, Dumont effectue une rupture décisive avec le point de vue matérialiste réducteur et mécaniste qui nie jusqu'à la réalité même de cet aspect du monde social et qu'illustre, parmi d'autres, Gerald Berreman: "Le monde indien de la hiérarchie rituelle que décrit Dumont est aussi stérile et irréel que le monde de la stratification dépeint par les sociologues qu'il critique ${ }^{2}$.»

I - Cf. L. Dumont, Homo hierarchicus, en particulier p. 15, 16, 57 et 58.

2 - G. D. Berreman, "The Brahmanical View of Caste », Contributions to Indion Sociology, numéro spécial, 5, décembre 1971, p. 18 (je souligne). 
Si cette rencontre peut se faire sur le mode diune double méconnaissance, tant du point de vue de sa production que de sa réception. c'est parce qu'elle implique, entre autres choses, un processus de transformation cognitif qui tient au travail de déplacement et de mise en forme effectué par Dumont entre ces deux espaces intellectuels, sans qu'il soit nécessaire d’en faire le résultat d'un projet conscient. En effet, en faisant de la sociologie des valeurs de Weber une caution de départ plus quine référence théorique, puisqu'il concède ultérieurement que sa "recherche se situe volontairement hors du paradigme weberien ${ }^{31}$ ". en parlant d'idées et de valeurs au lieu de principes transcendants, en glissant de la notion durkheimienne de totalité sociale à celle, métaphysique, de "Tout universel ", en citant Alexandre Koyré ou Thomas Kuhn à propos de la "crise du paradigme idéologique moderne ${ }^{32}$ ", et non René Guénon, bref, en replaçant une thématique marquée et datée sur la tradition et la crise du monde moderne dans un autre système de références théoriques explicites, Dumont opère, pour lui-même et pour son lecteur, un changement d'univers mental qui appelle une posture académique pour débattre d’une thématique idéologique, interdisant par là même de la rapporter immédiatement et directement à l'espace intellectuel et social dont elle dérive. Dumont contribue ainsi à faire de son ceuvre une structure de compromis, au sens analytique, toujours susceptible d'unc double lecture selon lunivers auquel on la rapporte, sans pouvoir cependant être réduite à l'un ou lautre de ces deux espaces ${ }^{33}$.

\section{THÉORIE INDIGÈNE ET MODĖLE SOCIOLOGIQUE}

L'un des obstacles épistémologiques auquel se heurte la compréhension sociologique de l'hindouisme est ce que l'anthropologue T. N. Madan appelle élégamment le home made model. De manière générale, le sociologue doit toujours prendre en compte deux ordres de compréhension du monde social: d'une part, la connaissance indigène et, d'autre part, la connaissance savante, quil faut construire pour rendre compte du point de vue indigène. Mais la difficulté est accrue lorsque le sociologue est confronté à une compréhension indigène de type savant, dont la culture hindoue fournit un paradigme particulièrement dense bien qu'il ne soit pas une exception ${ }^{3 \cdot 4}$ : on ne peut en effet oublier que thindouisme classique possède son modèle savant interne. qui est le produit du système lettré indigène, cest-à-dire brahmanique ${ }^{35}$. Or, tout se passe comme si Dumont ne distinguait pas ces deux niveaux ou, plus exactement, comme si la distinction qu'il opérait restait prise dans la compréhension indigène: il y a. écrit Dumont, "deux modèles indigènes, dont l'un est très ancien [le modèle des larna] mais toujours présent dans la culture indépendamment de mon interprétation. [...] Je propose de le reconnaître comme étant sousjacent et complétant lidéologie du système des jati ${ }^{36}$ ". Mais en se donnant pour tâche de "systématiser la thé(orie indigène [...] des castes ${ }^{3^{-}}$". Dumont réintroduit alors ce qu il avait congédié à l'origine de son projet : le point de vue lettré brahmanique, qu il présente comme le modèle sociologicue savant du système des jati. Ians ces conditions, tout se passe comme si le travail dinterprétation à l'ceuvre dans Homo hierarchicus était un véritable processus de transsubstantiation épistémologique au cours duquel Dumont, prenant les valeurs du brahmanisme comme principe de vérité scientifique du monde social hindou et non comme objet d’étude, transmuait malgré lui en théoric savante la théorie lettrée indigène du système des castes retraduite en langage sociologique 38 .

31 - L. I)umont. Essats sur lindit idualisme. of), cit. p. 23 (je souligne).

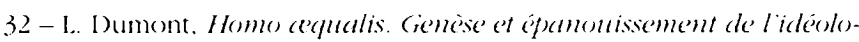
gie contemporaine. Paris. Gallimard. 19-7 p. 18.

33 - Cf. P. Bourdien, I. ()melegie pelitique de . Hartin Heidegger Paris Ed. de Minuit, 1988, à qui jemprunte ce schema dintelligibilite

$3 . t$ - Le rapprochement impose en effet arec la theoric medievale des trois ordres qui se fonde précisément sur la tripartition indo-eurepéenne des fonctions entre les prêtes. les guerriers et les paysans: (f. G. Duby, les Trois Ordres on lTmaginaire du féolalisme. Paris. Gallimard. 1978. Mais la spécificité de monde hindou réside dans la position de monopole ketré que détient la classé des brahmanes. et dont les effets de connaissance sont inscrits dans la thécrie incligene des castes. Je reviendrai dans un travail en préparation sur cet aspect essentiel a la comprehension sociologique de l'hindouisme.

35 - De ce point de vue, il me semble que lat distinction courante que fait lanthropologie anglo-saxonne entre systemes de significations locales (de type "emic") el interprétations externes (de type "etic") ne suffit pas à rendre compte des problèmes épistémologiques qui sont étucliés ici.

36 - L. Dumont, "On Putative Hierarchy and Some Allergies to It ". contributions to Indian Sociologl: numéro spécial. 5. décembre 1971 p. 73. La société brahmanique est divisée en quatre tarna (ou classes) ordonnés hiérarchiquement d dune part. les brahmanes (prêtres). les ksatrija (guerriers) et les iatsial (agriculteurs et commersants), qui ont acces au sacrifice védique, et, dautre part les sudra lau service des trois autres classes), qui sont exclus du sacrifice. Le terme jati (littéralement : naissance) désigne les castes que lon observe sur le terrain.

37- L. 1)umont. Homo bierarchicus, op. cit. p. 56 (je souligne).

38 - Dumont opère ici un glissement du se'ns logique de l'hindouisme comme système de valeurs à son interprétation cialuatice. pour reprendre les expressions de Weber. On retrouve en outre la polémique qui opposia Durkheim a lorientaliste James Darmesteter. ce dernier voulant faire du sentiment religieux " le critere de la verité scientifique" et non le considérer comme "objet de science". cf. E. I urkheim. Les Regles de la methode sociolegique. Paris. Flammatrion, 1988. p. $126-12^{-}$ 


\section{LE REFOULEMENT DE LA SCIENCE INDIGÈNE}

Dans les années qui suivent la fondation, en 1957, par Louis Dumont (en collaboration avec David Pocock) de la revue Contributions to Indian Sociology, un débat s'instaure sur la nature de la sociologie que Dumont entend fonder: s'agit-il de développer une sociologie indienne, comme le titre de la revue le laisse entendre, ou s'agit-il de faire une sociologie du monde indien, comme l'indique le texte inaugural de Dumont, «For a Sociology of India' », qui ouvre le premier numéro de la revue? La critique est d'abord formulée par l'anthropologue britannique F. G. Bailey, qui défend un point de vue de type objectivant; il reproche à Dumont de réduire le monde indien aux valeurs de l'hindouisme et s'interroge: existe-t-il une sociologie indienne comme il existerait « une chimie indienne ${ }^{2}$ » ? Pour sa part, l'anthropologue indien A. K. Saran reproche à Dumont de ne pas développer une véritable sociologie indienne, puisque son projet ne s'accorde pas avec la théorie brahmanique de la connaissance, fondée, selon A. K. Saran, sur une sorte de monisme idéaliste ${ }^{3}$. Face à ces critiques, la réponse de
Dumont est claire: «Une sociologie hindoue est une contradiction dans les termes [...]. La sociologie est une dans son principe. [...] II n'y aura jamais deux sociologies, laissez de côté une sociologie de type solipsiste. Je ne doute pas que le système des castes soit un type d'expérience sociale important qui réserve des leçons à la sociologie comme science, mais cela demande que ses enseignements soient traduits dans le langage universel de la sociologie ${ }^{4}$. »

1 - «For a Sociology of India », Contributions to Indian Sociology, I, avril 1957, p. 7-22; trad. de Louis Dumont, «Pour une sociologie de l'Inde », op. cit., Paris, Armand Colin, 1964, p. 89-II3.

2 - F. G. Bailey, «For a Sociology of India? », Contributions to Indian Sociology, III, 1959, p. 97 (souligné par Bailey).

3 - A. K. Saran, (Review of) Contributions to Indian Sociology, IV, 1959, in The Eastern Anthropologist, vol. XV, I, 1962, p. 53-68.

4 - L. Dumont, "A Fundamental Problem in the Sociology of Caste », Contributions to Indian Sociology, IX, décembre 1966, p. 23 et 24 (souligné par Dumont).

\section{«HOMO HIERARCHICUS 》: UNE LECTURE «DHARMIQUE » DE L'HINDOUISME}

Le travail d'alchimie cognitive que Dumont fait subir à la théorie brahmanique du système des castes se cristallise dans les notions de hiérarchie et de pouvoir, dont on retiendra trois aspects étroitement imbriqués. Premièrement, Dumont donne à la notion de hiérarchie un sens "purement religieux" : c'est ce qu'il appelle la "hiérarchie proprement dite " ou encore la " hiérarchie pure "39; deuxièmement, Dumont restreint la notion de pouvoir "exclusivement au pouvoir politique ${ }^{40}$ " ; troisièmement enfin, il donne un essai de contenu formel, logique, à ce qu'il dégage comme un invariant, la relation hiérarchique d'englobement.

La distinction radicale que Dumont opère entre hiérarchie et pouvoir veut rendre compte d'un fait central de l'hindouisme : dans cet univers structuré par l'opposition du pur et de l'impur, le prêtre, en terme rituel, est supérieur au roi; ou, si l'on veut, les valeurs religieuses, rituelles, "englobent " les valeurs du politique, du pouvoir, qui ne sont pas autonomes. Cette supériorité s'observe d'abord dans le monde social: les castes dominantes, qui détiennent la terre et reproduisent localement la fonction royale, ont un statut rituel inférieur à celui des brahmanes. En outre, ajoute Dumont, "Il y a identité entre supériorité et pureté supérieure; c'est en ce sens qu'idéologiquement la distinction de pureté fonde le statut ${ }^{41}$. "En d'autres termes, ce n'est pas le pouvoir ou la richesse qui fonde le statut dans le monde hindou. Reprenant la distinction esquissée par Weber entre groupes de statut et classes à fondement économique, on pourrait interpréter cette opposition en terme d'indépendance relative de principes de classement concurrents mais complémentaires ${ }^{42}$. Mais cette interprétation se heurte à la lecture que Dumont propose de la théorie lettrée indigène des varna, selon laquelle les deux principes de classement, rituel et social, seraient absolument distincts et hiérarchisés. Ainsi, l'hindouisme opérerait une disjonction radicale entre le varna des brahmanes et celui des ksatriya et, de manière homologue, entre les valeurs cardinales associées à chacune de ces classes : d'une part, le statut, qu'exprime la notion de dharma, et, d'autre part, le pouvoir économique et politique, qu'exprime la notion d'artha (intérêt) et qui

39 - L. Dumont, Homo bierarchicus, op. cit., p. 93 et 103.

40 - Ibid., p. 105.

41 - Ibid., p. 80. Pour une vue synthétique, cf. R. Deliège, Le Système des castes, Paris, PLF, 1993.

42 - Dumont esquisse parfois ce type danalyse en montrant que le don aux brahmanes est "un moyen de transformation des biens matériels en valeurs "; cf. L. Dumont, "Caste, racisme et "stratification" ", in Homo hierarchicus, op. cit., en particulier p. 318 
serait subordonné au dharma du point de vue des valeurs religieuses ${ }^{+3}$.

Des critiques érudites ont été apportées à ces analyses, mais elles ont en commun de laisser en suspens toute interrogation sur la théorie de la connaissance implicite que Dumont engage dans la construction de son objet. Si l'idéologie, entendue comme système de valeurs, est le principe objectif qui fonde, selon Dumont, le système des castes, c'est parce que " le point de vue empirique est un contresens quant à la civilisation indienne: il revient à mettre le dharma dans l'artha $a^{44}$ ", c'est-à-dire à inverser l'ordre des relations hiérarchiques entre les termes qui sont "englobant "et "englobé "dans l'hindouisme. Mais dans ce cas, le contresens est de confondre, du point de vue logique, les deux univers distincts que sont, d'une part, la représentation textuelle que le brahmanisme propose de la réalité du monde social hindou et, d'autre part, la réalité empirique de cette représentation. Plus encore, le contresens est de fonder, du point de vue cognitif, la connaissance sociologique de l'hindouisme sur les principes de la science indigène, c'est-à-dire sur la théorie brahmanique de la connaissance que Dumont s'approprie ici implicitement pour construire son modèle savant.

Si le monde de l'artha est relativement dépendant des finalités du dharma dans l'ordre de la représentation lettrée indigène, cela ne renseigne pas, a priori, sur le sens des relations observées dans le monde social entre les faits empiriques qui relèvent de l'un et de l'autre domaines. Ce qui importe au sociologue, c'est de rendre raison de l'accord ou du désaccord qu'il observe entre, d'une part, les distributions des différentes espèces de ressources matérielles et symboliques entre les jati qu'il enregistre dans la réalité du monde empirique et, d'autre part, les structures cognitives des individus ou des groupes, selon que celles-ci sont ou non structurées par l'ordre de ces distributions objectives. Ce sont ces structures de pensée, ces systèmes de valeurs, qui s'expriment, entre autres, dans les discours indigènes dont les catégories n'appartiennent pas nécessairement à l'univers savant, bien que celui-ci impose ses principes de classement comme références légitimes, de manière différenciée, à l'ensemble du monde social.

Aussi le sociologue doit-il encore s'interroger sur les principes cognitifs qui sont au fondement même de la représentation indigène du monde social, au risque de se laisser imposer un point de vue particulier sur ce monde. En adoptant le point de vue prescriptif des traités juridico-religieux qui constituent le dharma en principe universel autour duquel se subordonnent toutes les autres fins de l'homme, Dumont fait une lecture de l'hindouisme savant que lion peut qualifier non seulement de brahmanique, mais plus justement encore de "dharmique ". Ce point de vue le conduit à dénier la réalité des rapports de domination dans le monde hindou, affirmant que le problème " nexiste comme tel que dans l'esprit de l'analyste ${ }^{45}$ ". En effet, d'un point de vue brahmanique, les relations qui divisent et opposent les castes ne peuvent faire sens comme rapports de domination. parce que les valeurs du dharma, en même temps qu'elles s'imposent universellement au monde hindou, fût-ce différentiellement selon les groupes, intègrent l'ensemble des castes dans une représentation unitaire et consensuelle du monde. Mais, comme le rappelle Charles Malamoud: "Quelque insistance que l'on mette, à juste titre, à souligner la différence entre hiérarchie et pouvoir, il est clair que, même en Inde, la hiérarchie n'a de sens que par la domination que le supérieur exerce sur l'inférieur. On peut s'interroger sur la nature de cette domination, prendre au sérieux les justifications qui en sont données, on ne peut en nier la réalité to."

En outre, on ne peut oublier que le brahmanisme. au moins tel qu'il s'exprime dans lespace des textes savants. est traversé par des oppositions internes qui ont pour enjeu. non seulement ce que doit être la "bonne" définition du dharma, mais aussi, plus généralement, la définition clu principe même de classement efficient dans cet univers. En dégageant un point de vue que lion peut qualifier de perspectiviste, Charles Malamoud montre quil y a entre les trois principales fins de thomme, i.e. ke dharma (l'ordre), l'artha (l'intérêt) et le kama (le désir), une hiérarchie non pas absolue mais relative ou, selon son expression, une "hiérarchie tournante", qui varie sclon le point de vue de l'Ordre, de l'Intérêt ou du Désir que ces textes prennent sur les buts de l'homme ${ }^{+7}$.

43 - Pour une vue détailké of. I. Dumont. "La conception de la royauté dans l'Inde ancienne" in Home bierarchicus, of cit.. p. 351 375. Le dharma, ici l'orde socio-cosmique, constitue avec l'artha (l'intérêt) et le kama (le désir) lés trois fins de lhomme (purushartha) dans thindouisme classique: of. M. Biardeau. LFindowisme. Anthropologie dune civilisation. Paris, Flammarion. 1981, en particulier p. 40-76.

44 - L. Dumont, Homo hierarchicus, of cit. note 71a. p. 195.

15 - L. Dumont. Homo hierarchicas. (p) cit.. note $118 \mathrm{~g}$. p. 298 . Sans "décrier " la hiérarchie. il est difficile de distinguer celle-ci des faits d'inégalité : Dumont note à juste titre que lhindouisme présente bien - une théerie générale de l'"inégalité" "en ce qu'il donne un sens à celle-ci : cf. ihid. p. 323

46 - C. Malamoud. "Le Malencontre de La Boćtic el les théories de linde ancienne sur la nature de la société "in .M. Abensour (éd.). l. Tsprit des lois santages. Pierre Clastres on une notwelle antbropologie politique Paris, F́d. du Seuil. 1987. P. 17 t. 175.

47 - Cf. C. Malamoud. "Sémantique et rhétoricque dans la hiérarchie hindoue des "buts de l'homme" ". in Cuire le monde. Rite et pensée dans linde ancienne. Paris. La decouserte. 1989. p. 137-161. 
L'opération de purification de la hiérarchie à laquelle Dumont procède. consiste à amputer celle-ci "de ce avec quoi [elle] est mélangée la plupart du temps, à savoir le pouvoir ${ }^{48}$ ", ćest-à-dire à lamputer de ses déterminations sociales, qui en font en quelque sorte une hiérarchie impure. Il semble donc spécieux de vouloir réduire la notion de hiérarchie à un simple principe intellectuel ou, micux, une "structure logique" dont la valeur de vérité serait indépendante de son univers de référence, comme le fait Dumont ${ }^{49}$. On ne saurait oublier, au risque d'offrir une interprétation particlle autant que partiale de l'hindouisme, les leçons que I)urkheim et Mauss tirent de leur travail sur les classifications primitives: "Bien loin que [...] ce soient les relations logiques des choses qui aient servi de base aux relations sociales des hommes, en réalité ce sont celles-ci qui ont servi de prototype à celles-là. [...] Les premières catégories logiques ont été des catégories sociales [...]. Ainsi, la biérarchie logique n'est qu'un autre aspect de la biérarchie sociale $1 . .\left.\right|^{50}$."

\section{L'HINDOUISME ET L'UNIVERSEL}

Pour comprendre pleinement la place que la science indigène occupe dans l'anthropologie de I umont, il faut encore interroger le statut cognitif des principes égalitaire et hiérarchique qui fondent son projet de sociologie comparée. Ces principes, affirme-t-il, "sont des réalités premières" qui constituent deux "universaux ", c'est-à-dire deux exemplifications de "l'essence de l'homme" ". Mais Dumont ajoute: "L’idéal égalitaire [...] est artificiel: il représente [...] une négation volontaire dans un domaine restreint d'un phénomène universel ${ }^{52}$. "Peut-on alors considérer ces deux valeurs comme des réalisations équivalentes de "l'essence de l'homme"? Seule la hiérarchic pourrait être envisagée comme un "universel ", la valeur égalitaire n'ayant qu'une prétention culturellement et historiquement déterminée à ce titre. De fait, pour Dumont, "l'innovation moderne 53 "se dégage d'un fonds universel commun à toutes les sociétés, dont l'Inde représente un cas de figure exemplaire. Ainsi. écrit-il, la mise en évidence du principe hiérarchique qui structure cette formation sociale nous a "appris quelque chose sur la configuration de valeurs de type commun, non moderne, je suis tenté de dire normal ${ }^{5.1}$. Ft par un glissement de la notion de "totalité sociale "à celle de "Tout universel ", selon laquelle l'inférieur est englobé dans le supéricur, Dumont considère que "chaque configuration particulière d'idées et de valeurs est contenue avec toutes les autres dans une figure universelle dont elle est une expression partielle ${ }^{55}$. I)ans ces conditions, on com- prend que " le modèle moderne "soit pour Dumont " une variante exceptionnelle du modèle général et demeure enchâssé, ou englobé. à l'intérieur de ce modèle " ${ }^{56}$. Et balancant entre le langage descriptif et le langage prescriptif, il conclut que "la hiérarchie est universelle", ou mieux, que "la hiérarchic est une nécessité universelle " 5 ? Ainsi, tout se passe comme si I umont, qui reconnaît pleinement les déterminations historiques et culturelles de la valeur égalitaire, déniait ces propriétés au principe hiérarchique qui représente alors, seul, "l'essence de l'homme" parce qu'il est comme enraciné "dans la nature des $\operatorname{choses}^{58}$ ".

Mais ce projet souleve encore un second ordre de difficultés. Selon Dumont, il faut rompre avec le paradigme individualiste qui domine les sciences sociales, et fonder une sociologie comparative de type "holiste" sur les valeurs qui expriment, pour chaque univers culturel considéré, le point de vue englobant de l'universel ${ }^{59}$. Toutefois, on comprend difficilement comment cette démarche peut éviter de se récluire à la juxtaposition de sortes de solipsismes culturels résultant de labsolutisation, au mieux, de deux systèmes de valeurs incommensurables: soit, d'un côté, "l'universalisme individualiste" occidental et moderne qui rapporte toutes les cultures à laune de ses valeurs et, de lautre, le "holisme culturel" sur lequel se fonderait un point de vuce "différentiel" sur l'altéritée ${ }^{(0)}$. En enfermant le débat dans cette alternative

48 - L. Dumont. Homo bierarchicus, op. cit.. p. 269.

19)- (.f. L. Dumont, "Vers unc thérie de la hierarchie", in Homo bierar chicus. of cit. p. 396-403: et pour une vue theoriciste exemplatre. cf. S. Tcherkézoff. "la relation roi-prêtre en Inde sedon I.ouis Dumont. Le modèle de linversion hiérarchicque ". Circalbiea. 14. 1993, p. 65-85.

$50-\hat{\mathrm{E}}$. I urkheim et .1. Mauss, "1)e quelques formes primitives de classification. Contribution a létude des représentations collectives". in M. Mauss, limese 2. Paris. Éd de Minuit. 1968. p. 83 et 84. Pour comprendre pleinement lanthropologie de l'Inde de Iouis I )umont, il faudrait encore prendre en compte la vision du monde musulman cquil propese en opposant lislam égalitaire à thinclouisme hérarchique: pour une critique de ce point de vue. of. M. Gaboricau, Ni brabmanes ni ancêtres. Colpontenons musulmans du vépal. Nanterre. Société dethnologie, 1993.

51 - L. I)umont. Homo bierarchicus. op. cit. p. 15 et 23 (je souligne).

$52-$ Ibid. p. 33 et 34 .

53 - I. Dumont. Essais sur linditidualisme. (p). cit.. p. 23.

54 - Ihid.. p. 248 (je souligne).

55 - Ihid.. p. 258: sur la notion de "Tout universel. . of. p. 196.

$56-$ Ibid. p. 259 .

57 - L. Dumont. Homo biererchicus, op. cit., p. 300 (je souligne)

$58-$ L. 1)umont. Homo cepualis. op. cit.. p. 199.

59 - Cf. L. I)umont, " The individual as an Impediment to Sociological Comparison and Indian History ". in V. B. Singh et B. Singh (eds). Social and Economic Change : Esay's in Homnour of I). P. Hukberji. Bombay. Allied Publishers. 1967. p. 227-268.

6()$-\mathrm{L}$. 1)umone, Essais sur l'inditidualisme, (op. cit. p. $192 \mathrm{sq}$. 
radicale. Dumont ne peut que contraindre lanthropologue a transmuer ses choix méthodologiques en une prise de position d'ordre éthico-politique sur des valeurs ultimes, lui interdisant toute comparaison véritablement sociologique de la différence ${ }^{\text {gl }}$. I.e "holisme méthodologique " céfini par l umont semble alors ouvrir la voie à un relativisme généralisé des points de vue et, plus encore à la possibilité de justifier autant danthropologies qu il pourrait y avoir de systèmes de valeurs. Mais la position de I umont à cet égard n’a pas varié depuis les débats dés années 1960: "Il est impossible [...]. écrit-il, de subordonner tout à fait liuniversalisme [au holisme culturel] sans détruire lanthropologie, et on reléguera à la place qui leur revient les rêveries sur une multiplicité d'anthropologies correspondant à la multiplicité des cultures ${ }^{62}$."

\section{«SCIENCE FONDAMENTALE 》 ET « SCIENCE (CLASSIQUE) »}

Pour résoudre cette aporie, Dumont ne peut éviter de sinterroger sur la nature de luniversel qui est en jeu et, plus particulièrement, sur la raison comme principe de la posture scientifique. "Normativement, écrit-il, luniversel, cest lat rationalité. et les losis scientifiques sont communément données comme les seules propositions non-tautologiques vaiment universelles. Notre problème est de nous situer par rapport à cette rationalité-là (3.3. "Mais la critique du rationalisme et de la science que développe I)umont participe moins diune sociologie de la connaissance que de lessai philosophique empreint diune humeur idéologique "antimoderne" dans lequel les valeurs de "lindividualisme" et de la "modernité" sont opposées, globalement, aux valeurs "holistes" des sociétés traditionnelles, qui ont su préserver une vision harmonique du moncle en "subordonnant lhomme à lat totalité sociale ${ }^{\text {(xt }}$.

l.argumentation porte sur la dichotomie que la raison scientifique éablit entre les faits et les valeurs. En faisant une " distinction absolue entre sujet et objet ". en séparant de manière "illégitime" "être et clevoir être, faits et valeurs", "la pensée moderne" qui, en cela, "est exceptionnelle" 65 . constitue avec "lindividualisme ", dont elle est partite prenante. "lobstacle principal dans létude et la comprélension des sociétés non-modernes ${ }^{60} "$. Fn arrachant lhomme a lunite principielle de "lordre dans lequel les choses sont données" ". la science, qui "a une place. un rôle prédeminants dans lidéeslegie moderne ${ }^{\text {(x) }}$. brise la relation transcenclante que la tradition a éablie entre lhomme et lunivers. lélément et le tout. et elle abolit le sens du monde: "la destruction du cosmos hierarchicque ${ }^{(0)}$ "fait alors du monde moderne un monde dépourvu de valeurs. [... un monde soushumain. un monde diobjets. de choses. l... I un monde sans lihomme ${ }^{-1)}$.
De ce demi-constat nostalgique a la fois vague et flou ${ }^{-1}$, la conclusion s"impose pour Dumont: "Les idées modernes, scientifiques [...] étant liées au système moderne de valeurs. [...] sont souvent peu adaptées à l'étude de l'anthropologie et à la comparaison sociologique $^{-2}$. "Et puisque les sociétés traclitionnelles ne séparent pas les faits et les valeurs. "il ny a pas lieu dimposer cette complication ou cette distinction à des cultures qui ne la connaissent pas", on demeurera ainsi "plus pres de la relation réelle, dans les sociétés non-moderness" 73 . Dumont renverse alors les termes de lalternative et affirme que "le modèle comparatif fondamental doit être non-moderne $e^{-t}$,

Mais, contraint de concilier deux points de vue antinomiques, la prétention universaliste de la science et la critique radicale des valeurs qui la fondent, Dumont assigne à laanthropologie et, plus généralement, à la science sociale - si ce nést à la science en général - un programme imaginaire de restauration deun état primordial fantasmatique perdu. Ainsi écrit-il dans une vision prophétique: "On verrait bien plutôt la vocation de lanthropologie, en tant que science fondamentale, dans une démarche inverse et complémentaire de celle de la science (classique) et de lidéolongie moderne en général : ré-unir, com-prendre, re-constituer ce que lón a séparé. distingué. décomposé ${ }^{-5}$. " Lanthropologie accomplirait alors sa mission rédemptrice, qui est de "transcender" ke monde moderne. "ou plutôt de le réintégrer dans le monde plus humain que les sociétés avatient en commun jusque-là " ${ }^{76}$. Ainsi, par ce projet de restauration de son objet que Louis Dumont assigne à la connaissance, celle-

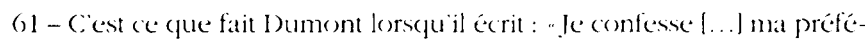

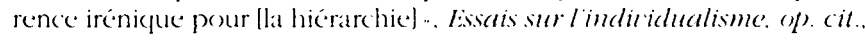
p. 26).

62-Ihid.. p. 199.

63 - Ibid. p. 207.

6.1-Ibid., p. 192.

$65-$ Ihid.. p. 221.

6() - Ihid. p. 2() 2$.

$6-5$ Ibid. p. 240.

(68-Ihid. p. 2.49)

(6) - Ibid. p. 208.

70 - Ibid. P. 255.

-1 - Sur la distinction entre fait et valeur che weber. of. C. Colliot

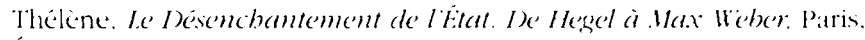
Éd. de . Minuit. 1992 .

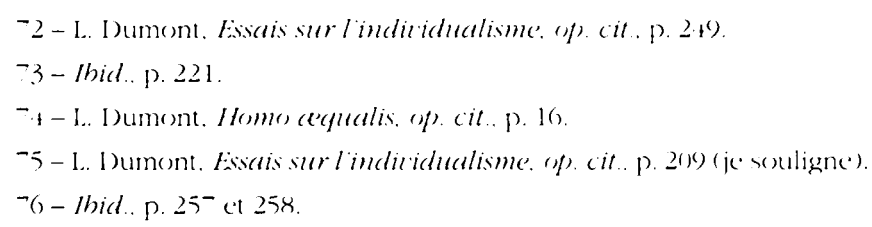




\title{
Ma génération, celle qui eut vingt ans autour de 1930 ou 1933*
}

\begin{abstract}
François Ewald : Pouvez-vous rappeler les principales étapes de votre itinéraire?
Louis Dumont: Cela commence par une révolte juvénile. À dix-huit ans, alors que je préparais l'École polytechnique au lycée Saint-Louis, j'ai brusquement décidé de tout laisser tomber. Ma mère, qui est veuve et a fait de grands sacrifices pour m'élever, me met à la porte. Cela devait être en 1929 ou 1930. J'ai été pris d'une espèce de révulsion devant l'espèce de vie bourgeoise qui s'offrait à moi. J'avais envie de vivre et de faire ce qui me plaisait.

J'ai vécu comme j'ai pu. J'ai travaillé comme employé d'assurance, puis comme correcteur d'imprimerie. Un beau jour, en 1936, quand j'étais au chômage, Georges-Henri Rivière m'a pris au musée des Arts et Traditions populaires pour coller des photographies. Je suis bientôt devenu secrétaire dactylographe du musée parce que je savais taper à la machine. Et là, j’ai décidé de reprendre mes études. Mon itinéraire relève donc du hasard le plus total. [...]

F. E. : Vous étiez d'une famille plutôt bourgeoise?

L. D. : Laborieuse et bourgeoise. Mon grand-père a été un des principaux dessinateurs de papiers peints du Second Empire et du commencement de la Troisième République. II a été aussi dessinateur de cachemires. Il a terminé sa vie professeur à l'école Bernard-Palissy. Et mon père, ingénieur de Centrale, a passé une grande partie de sa vie à diriger une compagnie de chemin de fer française de Salonique à Constantinople. [...]

F. E. : $\dot{A}$ la fin des années 1930, vous reprenez vos études.

L. D. : J'ai recommencé des études, une licence que je n'ai pu terminer qu'après la guerre. J'ai d'abord passé le certificat d'ethnologie en 1938. L'année suivante en 1939 je suis passé à l'École du Louvre, avec l'intention de faire plus tard une thèse d'histoire de l'art sur les survivances celtiques dans l'outillage artisanal contemporain. La guerre a éclaté et j'ai été prisonnier cinq ans'.
\end{abstract}

ci se trouverait réconciliée avec une science dite fondamentale ou, pour utiliser le langage plus adéquat ici de René Guénon, la "science profane" serait enfin réintégrée dans la "science traditionnelle", qui n'est autre que la "science sacrée".

\section{Censure et travail de mise en forme}

En se fondant sur les éléments biographiques rendus publics, on peut tenter d'objectiver la trajectoire de Dumont afin de rendre compte des choix successifs qui ont orienté ses investissements scientifiques. On peut ainsi espérer rendre compréhensibles les conditions qui ont favorisé le travail de conversion en termes académiques légitimes d'interrogations d'ordre idéologique développées, dans less années 1930, aux pôles extrêmes du champ intellectuel et politique. Les contraintes des rapports de force spécifiques liés à la position institutionnelle que Dumont occupe dans l'espace des disciplines, à l'intersection des études indiennes classiques et de l'an-

\footnotetext{
* «Dumont l'intouchable» (entretien avec Jean-Paul Enthoven), Le Nouvel Observateur, 6 janvier 1984.

1 - «Louis Dumont, la culture de l'individualisme » (propos recueillis par François Ewald), Les Nouvelles Littéraires, octobre 1991, p. I14-119.
}

thropologie sociale, lui imposent en effet un travail de mise en forme qui agit comme un véritable effet de censure, lui interdisant de dire et de se dire complètement ce que sa vision sociologique de l'Inde doit à la part la plus refoulée de l'histoire de l'univers dont il participe. Mais en mettant en relation la genèse de l'œuvre avec la trajectoire de Dumont, on peut aussi s'interroger sur les conditions intellectuelles et sociales qui ont permis, pour une part, une levée progressive de ces censures et facilité ainsi un retour de thématiques antérieurement refoulées. En effet, tout se passe comme si la résurgence des interrogations de type guénonien, jusque dans leurs modalités expressives, devenait d'autant plus manifeste que le propos de Dumont est suffisamment transformé en généralités d'ordre socio-philosophique et éloigné, avec le temps, de la période où ces préoccupations ont été refoulées.

La première période "commence par une révolte juvénile "au début des années 1930, lorsque Dumont est pris d'une sorte "de révulsion devant la vie bourgeoise" qui s'offre à lui. Elle est marquée par une double rupture, 
sociale et scolaire, avec le projet familial (maternel) qui le destinait à l'École polytechnique, selon le modèle de reproduction que le fils unique a hérité du père décédé dans la petite enfance. Cette rupture est à la fois brutale et douloureuse : sa mère lui fait "un véritable esclandre " et le "met à la porte". Suivent des années difficiles où alternent chômage et périodes de petits travaux. Mais ce sont aussi des années de formation au cours desquelles Dumont "s'imprègne" des idées de Guénon, alors qu'il "se meut sur les marges, entre un existentialisme à lancienne mode et le surréalisme " ${ }^{77}$.

Une deuxième période débute avec la rencontre de George-Henri Rivière et l'entrée de Dumont au musée des Arts et Traditions populaires "tout à fait par hasard ", "par une toute petite porte "; engagé d'abord "pour coller des photographies", il devient ensuite "secrétairedactylographe". Si les conditions contingentes de ses débuts et les ambitions alors modestes de Dumont contrastent avec l'importance qu'il attribue rétrospectivement à ces années d'apprentissage, c'est parce que celles-ci marquent les vrais commencements où s'enracine, objectivement et subjectivement, sa trajectoire académique, orientée d'abord vers l'histoire de l'art et le monde celte avant d'être dirigée vers l'anthropologie de l'Inde: "Tout commence en 1936-1937", dit Dumont, c'est-à-dire en cés années et en ce lieu où, rétrospectivement, il se "découvre, en somme, une vocation d'ethnologue "à travers les activités du musée, l'enseignement de Marcel Mauss et, aussi, en la personne de Claude LéviStrauss, alors jeune agrégé de philosophie de retour du Brésil préparant son ouvrage sur la parenté. On peut faire l'hypothèse que ce dernier a représenté la figure exemplaire du savant à laquelle Dumont s'est identifié au début de sa carrière d'anthropologue autodidacte, permettant ainsi la cristallisation de sa propre vocation ${ }^{78}$.

Mais ce sont probablement la Seconde Guerre mondiale, les années passées comme prisonnier et l'apprentissage du sanscrit que Dumont fait alors qui marquent la rupture la plus décisive avec la période de formation d'avant-guerre, abandonnée dès lors à l'oubli sélectif de la mémoire. De retour de captivité, Dumont s'oriente définitivement vers l'étude du monde indien et la vie académique. Dans les années d'immédiat après-guerre, il effectue un travail de terrain en Inde du Sud. Après avoir enseigné quelques années à Oxford où il parfait sa formation au contact des maîtres de laanthropologie britannique, il est nommé en 1955 directeur d'études à la $\mathrm{VI}^{\circ}$ section de l'École pratique des hautes études, poste qu il occupe jusqu’à sa retraite.

Les recherches que Dumont conduit jusqu à la fin des années 1950 constituent la première partie essentielle- ment ethnographique de son ceuvre. L'étude de la Tarasque, fête populaire du sud de la France, la monographie consacrée à la sous-caste des Pramalai-Kallar en Inde du Sud et les recherches sur la parenté apparaissent, selon les termes de Dumont, comme autant de "publications plus techniques", fortement marquées par les orientations méthodologiques qui prévalaient à cette époque dans l'univers anthropologique français: privilège accordé à l'approche de type monographique, importance de la culture matérielle. point de vue strictement structuraliste dans létude de la parenté sont autant d'éléments qui permettent à Dumont de fonder un savoir ethnographique sur une expérience de terrain, avant d'élaborer son modèle de compréhension du monde indien.

Homo bierarchicus constitue la seconde partie de l'ceuvre, marquée par le passage d'un point de vue ethnographique local à un point de vue sociologique général sur le monde indien. Aussi ce travail engage-t-il un processus nouveau de mise en forme conceptuelle du savoir qui se prête plus aisément que les recherches précédentes au retour des schèmes mentaux hérités de la lecture de Guénon. Mais les conditions intellectuelles et sociales qui président au travail de Dumont, interdisent cependant que la vision guénonienne de l'Inde et du monde moderne s'exprime directement dans Homo hierarchicus: non seulement paree que les savoirs anthropologiques sur lesquels ce travail se fonde, ne participent pas de l'univers guénonien, mais surtout parce que cet ouvrage élaboré dans les années 1960) sinscrit dans une institution académique situéc au pôle le plus légitime de la recherche en sciences sociales, et clans une conjoncture intellectuelle dominée à la fois par la prévalence du paradigme structuraliste et l'importance de travaux diversement influencés par le marxisme. Plus précisément, alors que Dumont n'a cessé de critiquer les "vues superficielles" que "la perspective matérialiste de la société et de lhistoire "79 présente de la civilisation indienne, tout se passe comme si le point de vue structu-

\footnotetext{
77 - L. I) umont. "On the Comparative Linderstanding of Von-.Moclern Civilizations ", Daedalus, printemps 1975. p. 166. Dans les annees 1930. Guénon est lu a la fois pai les tenants de la droite nationaliste. comme Drieu la Rochelle. et par des intellectuels proches du parti communiste. par exemple Raymond Queneau.

78 - Outre que Clande Ievi-Strauss est le seul anthropologue contemporain de Louis l)umont. donc aussi le concurrent. régulièrement evorqué dans ses entretiens. cest aussi celui arec lecpuel tout traval de célébration. aujourd hui encore. le met ke plus frécfuemment en parallek lorsqu il sagit de dresser les a portrats médiatiques a des maitres de l'anthropologic francaise.

79- 1. Dumont. La Cirilisation indienne et Nons. Paris. Armand Colin. 196.t. p. 76 .
} 
raliste qu'il défend lui permettait de s'affranchir en esprit "aussi bien de lidéalisme que du matérialisme ${ }^{80}$ ", par une sorte de dépassement imaginaire de cette antinomie, favorisant ainsi la retraduction d'une vision essentialiste du monde social en termes épistémologiques apparemment neutres. Ainsi, l'ensemble des déterminations externes, constitutives de l'espace social spécifique dans lequel se développe l'anthropologie dumontienne, contribue à rendre irrecevable, du point de vue scientifique, toute référence à l'un des penseurs de la tradition les moins légitimes du seul point de vue académique.

C'est dans ce contexte qu'il faut comprendre lusage que Dumont fait de Tocqueville dans Homo bierarchicus. La référence à cet auteur clont la légitimité académique était encore faible au début des années 1960, en particulier auprès des sociologues se réclamant de l'école clurkheimienne, apparaît en effet sinon paradoxale, au moins en discordance avec l'espace épistémologique auquel Dumont se réfère: "Jétais un ethnologue, je me réclamais de Mauss et dautres durkheimiens, or Tocqueville était ignoré des sociologues comme des ethnologues. D'où diable avait pu me venir l'idée d'avoir recours à Tocqueville? Comment l'avais-je découvert ${ }^{81}$ ?" Que le travail de Raymond Aron ait "fort probablement " fourni le moyen de cette découverte ne renseigne pas sur la place à la fois essentielle et marginale que Tocqueville occupe dans l'économie du projet dumontien. De ce point de vue, on peut faire l'hypothèse que l'xeuvre de Tocqueville constitue la médiation nécessaire pour que soit reformulé en termes académiques légitimes, le projet de sociologie comparée entre les sociétés traditionnelles et le monde moderne que Dumont a hérité de Guénon.

Le rapprochement entre les thématiques développées par Tocqueville et par Guénon s'impose en effet pour plusieurs raisons. D'une part, à un siècle dintervalle, ces deux auteurs ont développé, chacun à sa manière, une ceuvre marquée par le spectre de la Révolution française et la nostalgie de l'Ancien Régime. Ils partagent ensuite, pour une part, une philosophie de l'histoire occidentale et une vision du monde social homologues, produites à partir des mêmes schèmes binaires clans lesquels s'opposent l' "ancien" et le "nouveau". la "tradition" et la "modernité", la "hiérarchie" et l'égalitée, l" aristocratie " et la "démocratie", les "castes" et les "classes", l' "élite " et le "peuple ", etc., permettant d'engendrer sans fin sur le monde social, en les combinant (la "démocratie " et la "caste" par exemple), les mêmes topoi dont les effets ressortissent plus de l'ordre de la rhétorique politique et journalistique que de l'ordre de la connaissance.

Ainsi, le thème tocquevillien de la montée de l'individualisme et du déclin corrélatif des solidarités dordre ancien dans la France moderne. par lequel Dumont ouvre Homo hierarchicus, traduit dabord, par ses modalités expressives, les réactions que l'on ose à peine clire aristocratiques de toute élite déchue de ses prérogatives politiques et symboliques. et qui se trouve en porte à faux dans un monde social ne reconnaissant plus les valeurs dexcellence qui la constituent dans son être même: l'effroi suscité par le mélange cles classes (ou des castes) autrefois " fort distinctes et immobiles ", la crainte "que les conditions s"égalisent " et lobsession corrélative du "nivellement " 82 , sont autant de fantasmes sociaux, parmi dautres, que l'on retrouve exprimés par Guénon dans la Crise du monde moderne, mais sur le mode inspiré. donc illégitime.

Enfin, Tocqueville et Guénon ont encore en commun une vision du monde qui se fonde, selon les modalités qui leur sont propres, sur un point de vue comparatif, sur une mise en perspective de la tradition (la hiérarchie des castes, de type aristocratique) au regard de la modernité (l'égalité démocratique) : l'Inde représentant chez l'un le passé "normal" de l'humanité, l'Amérique étant pour lautre le futur cle la démocratie qu'il contribue pour sa part à faire advenir (à la différence de celuilà). Aussi est-il paradoxal de voir Dumont établir sur ce point une filiation entre Tocqueville et Mauss, alors qu'il reconnaît précédemment lhétérogénéité des univers intellectuels auxquels ces deux auteurs appartiennent : "Ce qu'on appellerait la démarche en miroir de Tocqueville, écrit Dumont, a bel et bien préfiguré la nôtre telle que nous l'avons puiséce essentiellement dans l'enseignement de Mauss ${ }^{83}$. Mais lorsqu'on connaît l'opprobre qui frappait Guénon clans les milicux indianistes savants des années 1950 - "cet homme qui a introduit le ton de Gringoire dans la métaphysique ${ }^{\text {t. }}$ "-, on comprend que lunivers de pensée de Tocqueville ait pu simposer alors presque spontanément à Dumont et lui offrir ainsi une médiation indispensable pour exprimer en des termes acceptables et reconnus par l'univers savant, ce que les censures du champ lui interdisaient de formuler.

Mais c'est dans les travaux ultérieurs consacrés à la genèse de l'individualisme moderne que l'on voit ressurgir de manière à la fois plus diffuse et moins euphémisée,

80)- L. Dumont. Home hicrarchicus. of) cit. note 1a, p. 16.

$81-1.1$ )umont. "lexpueville et le respect de laute". Eyprit. 129-130). acrit-septembre 1987. p. 2. 3.

82 - Cf. les extrats de De la démocratie en Amerique de A de Tocqueville. cités par L. I)umont dans llomo bierarcbicus. op. cit., p. 32,33 .

83 - L. Dumont. "Tocqueville et le respect de lautre". Iox. cit. p. 3.

84 - Cité in Rene Guenon et liatualite de la pensée traditionnelle. Actes du colleque international de Cerisy-la-Salle (1973). Milan. Arche, 1980. p. 52. 
les thématiques aux raisonnances guénoniennes sur la science et la raison. par ailleurs étrangères à Tocqueville. Ces recherches constituent un troisieme ensemble dans l'ceuvre de Dumont, non seulement parce quelles ressortissent à une histoire des idées occidentales plus quà un travail empirique de type socio-historique, mais aussi parce quielles marquent son abandon brutal des études indiennes dans les années qui suivent les événements de mai 1968. Tout se passe comme si cette dernière rupture saccompagnait alors dun retour progressif de Dumont aux interrogations de sa jeunesse, que favorisent en outre l'évolution du champ de production culturelle et la reconnaissance quil y trouve alors.

Si l'oxuvre de Dumont est le résultat diune logique de recherche autonome qui ne doit rien aux effets de mode du marché des biens culturels, on ne peut comprendre l'intérêt que ses travaux sur lindividualisme et la modernité ont suscité dans des milieux intellectuels fort éloignés de la recherche indianiste sans prendre en compte un état nouveau dés débats idéologiques qui se sont imposés sur ce marché à partir de la fin des années 1970). L'émergence diune nouvelle catégorie de producteurs de biens symboliques, situés à lintersection du champ universitaire et du champ journalistique, sest accompagnée d'un renversement des thématiques qui définissent l'humeur idéologique ayant cours dans le champ intellectuel $^{85}$. Issus dans leur majorité des disciplines littéraire et philosephique mais partageant nombre de valeurs proches du pôle du pouvoir économique et politique dont ils participent également, ces nouveaux intermédiaires culturels ont développé une critique globale de ce quils nomment "la pensée $68^{86}$ ". Sopposant à la "philosophie des structures "et, plus généralement, à l'ensemble des sciences sociales réduites à lapplication de quelques schèmes issus du marxisme et du paradigme structuraliste. ces essayistes prônent la restauration des thèmes éternels cle la tradition philosophique spiritualiste, ceux de thumanisme et de la morale rou de léthique), de l'individu et du sujet. dont ils font les " nouveaux " créateurs de valeurs. Dans ce contexte. la multiplication dans les années 1980 de divers ouvrages, conférences. colloques et débats consacrés au thème de "l'individualisme" par les nombreuses instances de médiation culturelle qui assurent la libre circulation des idées dans le champ intellectuel (Collège international de philosophie ${ }^{x^{-}}$. Fspace séminaire du Centre GeorgesPompidou ${ }^{\text {xs }}$, revues Esprit ${ }^{x)}$ et le déhat. tribunes des grands quotidiens et des hehdomadaires. par exemple). est venue amplifier les travaux de Louis Dumont, qui apportait sur ce sujet sa caution d'universitaire érudit. Fn outre. la critique des formes instrumentales et technicpues de la raison (occidentale) que reprend Dumont rencontre des préoccupations diverses qui animent autant, d'un côté. les héritiers des pensées traditionnelles directement issues des années $1930^{\% 0}$ que, de l'autre, certains tenants de la philosophie analytique ou des courants de pensée " postmoderne ".

De nombreux ouvrages publiés dans les années 1980 , et qui sont de ce fait contemporains des Essais sur lindividualisme de Louis I Jumont. contribuent à définir cet air du temps marqué par le "retour" à un point de rue individualiste sur le monde social. qu'il s'agisse de Gilles Lipovetsky ${ }^{91}$. de Alain Minc ${ }^{92}$. de Luc Ferry et Alain Renaut ${ }^{93}$, ou de Alain Renaut ". La célébration de Inumont à laquelle participent ces auteurs, qui ignorent souvent ses travaux indianistes. en fait la référence incontournable sur le thème de lindividualisme, même sil est parfois sévèrement critiqué pour ses lacunes philosophiques ou ses partis pris traditionalistes. Ainsi Alain Renaut consacre le second chapitre de son ouvrage à "Louis Dumont ou le triomphe de l'individu ". et qualifie la "passionnante genèse de la modernité" de ce "magistral comparatiste" de "retour à Ithaque. terme dune extraordinaire odyssée de l'esprit "95. Mais dans le mème temps. le philossophe légitime rappelle à lanthropologue les lacunes de sa culture philosophique diautodi-

85 - sur les problemes evocpués ci-dessous. cf. en particulier L. Pinto, - Ia dexa intellectuelle "Actes de la recherche en sciences sociales. 90.

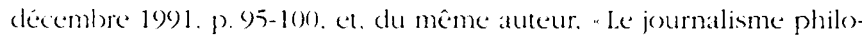
sophicpue.. Actes de la recherche en sciences sociales, 1011-102. mars 19). p. $25-38$

86 - L. Ferre et A. Renatu. Ia Pensée Ges. Essai sur lanti-hamanisme contemporain. Paris. Gallimard. 1985. reed. en coll. "Folio Essais. 1988. p. 16,17

87 - C. A. Renaut. "Sur lindividualisme "communication présentéce dans le cadre du college international de philosophie. Paris. 15 mai 1987.

$8 x$ - (f. 1). de Coppet. "The Sociely as an I'limate value and the socio-Cosmic configuration ". communication présentéc a lispace seminaire philosophice et anthropologice : "Relativisme-universalismeholisme-individualisme-hiérarchie ". Centre (eorges-pompidou. Paris. 2(6-28 arril 1980). Ethnos. 199(3. 3-4. p. 1.10)-150

8) - Cif par exemple le numéro de la revale Esprit de février 1978 , (consacré a louis D umont.

9()$-$ pour une interprétation des theses dumontiennes par la nouvelle droite. (f. P. Bérard. L. 1)umont: "Anthropologic et modernite . I.a Soncelle licole. 39. nowembre 1982. p. 95-115. Par ailleurs, lactualite de Guenon est attestée par la réedition en live de poxhe de la Crise du monde moderne. Paris. Crallimard. coll. "Folio Fisatis " 19).t. et par sa araduction. aujourd hui. dans les pays d'Europe de l'bst chongrie el Russic par exemple).

91 - (;. Liperelsky. I. Fre du ride. Fisatis sur lindividualisme contemprocain. Paris, Gallimard. 1983.

$92-$ A. Minc. La Hachine égalitaire Paris, (irasset. 198-

93 - L. Ferry el A. Renaut. (os-80, itineraires de l'inditidu. Paris. Gallimard. $198^{-}$.

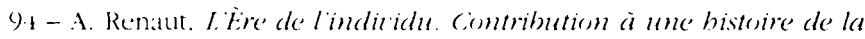
subjecticite Paris. (jallimard. 1989).

$05-$ Hoid. p $-14-3$. 
dacte ${ }^{90}$. tout comme il relève que "ladhésion antimoderne aux valeurs du holisme" de Dumont participe "de ces visions apocalyptiques de la moclernité que la pensée contemporaine ", selon Alain Renaut, affectionne particulièrement ${ }^{97}$. Larticle de Pierre Rosanvallon publié et intitulé "Louis Dumont, le sacre de lindividu 98" illustre la rhétorique politico-médiatique à laquelle se prête aisément la thématique dumontienne en période d'incertitude. La méthode comparative de Louis I Jumont " débouche sur une perspective philosophique ", puisquelle interroge "le principe de l'unité du genre humain": l'anthropologie est confrontée au "dilemme " entre le "relativisme" d'un côté et la "perspective universaliste "impérialiste" " de l'autre; "le cadre tracé par Dumont offre la possibilité " de repenser "lindividualisme et létatisme" autant que "les notions d'égalité et de justice", il travaille même "à sortir du keynésianisme et, plus globalement à réorienter la modernité ", il nous ramène, "en fin cle compte, à nos interrogations actuelles les plus pressantes " 99 .

Mais si l'on peut reconnaître à Louis Dumont le mérite davoir ramené dans le débat public des interrogations nées de ses recherches sur la civilisation indienne, la reconnaissance relative qu'il a trouvée dans l'élargissement de son audience ne va pas sans paradoxe. Le moindre des contresens que son oeuvre induit n'est-il pas en effet de faire de cet anthropologue le chantre de l'individualisme, alors même qu'il na jamais caché sa préférence pour les valeurs de la tradition, fûtce, comme le note Alain Renaut lui-même, au prix de

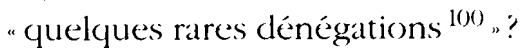

\footnotetext{
96 - Sur Fichte en particulier, cf. ibid.. p. 94.

97 - Ibid.. p. 91

98 - Libération, 17 novembre 1983, repris in Revue européenne des sciences sociales, XXII, op. cit.. p. 149-151.

99 - Ibid.. p. 151.

$100-$ A. Renaut, I. Tire de lindividu. op. cit.. p.91.
} 